\title{
Multi-hop transmission and routing with hierarchical modulation
}

\author{
Tae min Park , Joonsoo Lee, Dooyoung Kim and Chongkwon Kim
}

\begin{abstract}
Hierarchical modulation multiplexes layers of different robustness into one stream. Usually being exploited in broadcast systems such as digital video broadcasting, hierarchical modulation can also be applied to unicast communications in a cooperative manner. This article deals with novel multi-hop cooperative transmission and routing strategies with hierarchical modulation. In multi-hop networks, when intermediate nodes receive different sets of streams, they cooperatively forward streams toward the final destination. We first show how hierarchical modulation reduces the total number of transmissions in tandem networks. We then model an optimization problem that determines proper modulation schemes and routing strategies as a stationary infinite-horizon Markov decision process (MDP). The MDP problem requires exponential computations forcing us to delve for heuristic solutions. We simplify the optimization problem by restricting hierarchical modulation consisting of only two fixed constellations. Even with this approximation, the routing problem with hierarchical modulation is still too complex because we must consider all possible paths and hierarchical modulation schemes at the same time. We introduce an approximation scheme that determines the most efficient hierarchical modulation scheme for each three node subgraph assuming subgraphs are isolated each other. Based on the three node subgraph approximation, we map original graphs to virtual graphs. We then apply one of shortest path algorithm to the virtual graphs to find the best route with hierarchical modulation. We perform simulations with various network topologies to evaluate the efficiency of hierarchical modulation in multi-hop unicast communication. Our results show that hierarchical modulation enhances the throughput about 25-30\% compared to non-hierarchical modulation. However, optimal solution derived by solving the MDP is also 5\% better than heuristic solutions suggesting the necessity to develop better heuristics in future.
\end{abstract}

Keywords: Hierarchical modulation, Multi-hop network, Markov decision process, Heuristic metric, Routing metric

\section{Introduction}

In wireless networks, bandwidth is a scarce resource and should be efficiently used. Especially as the demands for mobile communications increase explosively, the importance of careful use of radio resources increases and the last decade has witnessed rigorous research on this topic. One approach that improves the efficiency of wireless networks is cooperative communication. In cooperative communication, multiple nodes collaborate to transfer information to final destinations.

Hierarchical modulation multiplexes layers of different robustness in one symbol. Receivers in good channel conditions can decode all layers including less robust layers

*Correspondence: tmpark@popeye.snu.ac.kr

School of Computer Science and Engineering, Seoul National University, Seoul 151-742, South Korea while receivers in bad reception conditions can decode the basic layer only. Originally hierarchical modulation was targeted to broadcast communication systems such as digital video broadcasting (DVB). In recent years, it has been also applied to simple unicast networks consist of a source, relay and final destination.

This article deals with unicast communications with hierarchical modulation in multi-hop wireless network. Most previous study that deals with unicasting with hierarchical modulation assumes networks of three nodesthat is a source, an intermediate node and a destination. This article lifts the restriction and investigates hierarchical modulation in general multi-hop wireless networks. In mesh network, we must handle routing as well as hierarchical modulation. The optimization problem is to find the most efficient end-to-end path that realizes the potential benefits of hierarchical modulation.

\section{是 Springer}

(c) 2012 Park et al: licensee Springer. This is an Open Access article distributed under the terms of the Creative Commons Attribution License (http://creativecommons.org/licenses/by/2.0), which permits unrestricted use, distribution, and reproduction in any medium, provided the original work is properly cited. 
As indicated in many previous studies, hierarchical modulation for unicasting induces cooperative communications. Two receivers, one close to a transmitter and another far from a transmitter, acquire different amounts of information. The receiver closer to the transmitter relays the additional information to the receiver farther away from the transmitter but closer to the final destination. The cooperative communications improve the network efficiency but make the routing problem complex because we need to consider hierarchical modulation with multiple nodes instead of two-node transmission in none-hierarchical modulation.

We model the optimization problem using a stationary infinite-horizon Markov decision process (MDP). An action in this MDP problem determines a transmitter and a hierarchical modulation scheme. A hierarchical modulation scheme decides basic and secondary constellations and the priority parameter $\lambda$. The optimization problem, modeled as an MDP, is not amenable to exhaustive search because the search space is very large. Therefore, we introduce several assumptions to make the problem attackable.

We first assume that all hierarchical modulation is $4 / \mathrm{m}$ QAM modulation. That is we assume that the basic layer is QPSK. This assumption is adopted in many previous studies and is reasonable in fairly well-connected wireless networks. We also assume that there are at most two receivers that accept signals from a transmitter. To reduce the solution space, we assume that two receivers are geographically closer to the destination than the transmitter. Also, the far receiver (receiver farther away from the transmitter) is closer to the destination than the near receiver. Even with these assumptions, the complexity of the MDP problem is still too high. If we use dynamic programming to solve the MDP problem, we get the exact solutions of the MDP only for very small networks.

The complexity of the MDP problem stems from the fact that it considers routing and hierarchical modulation at the same time. We devise a novel scheme that separates routing and hierarchical modulation. From the original graph, we pick a subgraph with a transmitter and two receivers. For each three node subnetwork, we select the best hierarchical modulation scheme that optimally transfers packets from the transmitter to the two receivers. After we find the best hierarchical modulation schemes for all possible subnets ${ }^{\mathrm{a}}$, we map a original network into a virtual network. Once virtual network mapping is completed, we then easily find the shortest path from a source to a final destination applying one of shortest path algorithms.

We carry out vigorous performance study using computer simulations. We use MATLAB for our study. First, to examine the effects of simplifying assumptions, we use small tandem networks with four or five nodes where the search for the optimal solutions is affordable. Our results show that hierarchical modulation enhances the throughput about 25-30\% compared to non-hierarchical modulation. However, optimal solution derived by solving the MDP is also 5\% better than heuristic solutions, suggesting the necessity to develop better heuristics in future.

\section{Related study}

Hierarchical modulation is a technique that can enhance the efficiency and flexibility of spectrum utilization. In hierarchical modulation, multiple data layers including a basic constellation and one or more secondary constellations are merged into a single stream [1]. Receivers in bad channel conditions can decode the basic layer only while nodes in good reception conditions can decode secondary layers as well. Therefore hierarchical modulation customizes transmission rates according to receivers' reception condition with one transmission.

Hierarchical modulation was originally targeted for broadcast systems. There are multiple receivers with different capabilities and requirements in broadcast and multicast systems. Pursley and Shea [2] proposed to use non-uniform modulation to provide customized transmission rates to different receivers according to their channel conditions. Jiang and Wilford [1] devised a backward compatible scheme to upgrade the performance of an existing broadcast system using hierarchical modulation. The basic constellation is the same as in the previous system and the secondary constellation carries the upgraded additional data. Hossain et al. [3] contrives a new technique for simultaneous voice and multiclass data transmission over fading channels using adaptive hierarchical modulation. More recently, a system that combines satellite and terrestrial communications using hierarchical modulation has been developed for distinguished deliveries of global and local information [4].

Hierarchical modulation has become an integral part of digital broadcasting systems (DBS) [5-7] such as digital video broadcasting-terrestrial (DVB-T), digital video broadcasting-handheld (DVB-H), MediaFLO and ultra mobile broadband (UMB). Recently terrestrial digital multimedia broadcasting (T-DMB) is upgraded to advanced terrestrial digital multimedia broadcasting (AT-DMB) [8] that employs hierarchical modulation. Owing to hierarchical modulation, AT-DMB can not only boost data rates but also can enjoy backward compatibility to T-DMB. In addition to standard or commercial broadcast systems, many researchers investigated the further enhancement of hierarchical modulation. Tamgnoue et al. [9] studied the performance of OFDM based hierarchical modulation integrated to DVB- $\{\mathrm{T}, \mathrm{H}\}$.

The error performance of hierarchical modulation has been rigorously investigated and exact BER expression 
over additive white Gaussian noise (AWGN) and fading channels are obtained [10]. Also, a recursive algorithm that compute the exact BER performance was introduces [11]. Chang and Lee [12] analyzed the BER of hierarchical modulation on cooperative communication systems. They also developed a criterion that determines the optimal distance parameters to guarantee certain levels of BER performance.

In addition to broadcast systems, several researchers [12-16] tried to adapt hierarchical modulation to unicast communications. Hausl and Hagenauer [14] first incorporated hierarchical modulation to a network that consists of one transmitter, one relay and one destination. Note that the three node network naturally forms a cooperative communication network because the relay node that acquires more data than the destination forwards the additional information. Lee and Cho [15] proposed a scheme that finds the best relay with hierarchical modulation. Whang, Huaping and Hong [17] analyzed the relationship between the distance parameters and symbol error rate performance and compare the performance of relay with hierarchical modulation with that of a conventional relay system. Similar study that determines the hierarchical modulation scheme at the relay node to optimize the performance depending on its SNR was also carried out [18]. Annamalai et al. [19] developed a technique that uses SNR to adapt rates at a symbol level in unknown time varying channels. Hucher and Sadeghi [13] found the optimal constellation parameter that minimizes error probabilities of packets for both relay and destination nodes when hierarchical modulation is used. An upgraded hierarchical modulation-based cooperation scheme using two relays allowing full duplex cooperative transmission was devised also [16].

\section{Hierarchical modulation and multi-hop transmission}

\section{Hierarchical modulation primer}

Figure 1 shows the constellation of 4/16-QAM hierarchical modulation. Two most significant bits of four constellation points in the same quadrant are the same, which makes most significant bits more robust than the least significant bits. In this case, the basic stream is encoded into two most significant bits while the secondary stream is encoded into remaining two bits. Even though a receiver with bad channel quality cannot fully decode all bits, it may decode two most significant bits by treating a received signal as a QPSK modulated symbol.

We can adjust the relative importance of the first and secondary information by adjusting two distance parameters shown in Figure 1 . One parameter is $2 d_{1}^{\prime}$, the minimum distance between any two symbols in different but adjacent quadrants. Another is $2 d_{2}$, the distance between two neighboring symbols within the same quadrant. Let

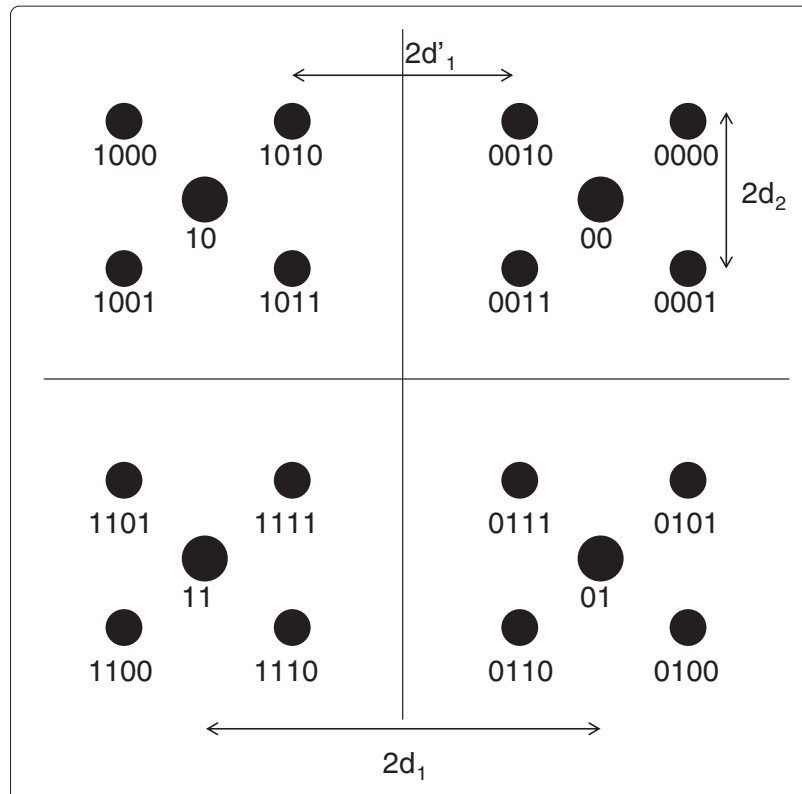

Figure 1 4/16 QAM hierarchical modulation.

$\lambda$ be $d_{2} / d_{1}^{\prime}$. The parameter $\lambda$ is constellation robustness parameter that determines relative robustness of basic layer and secondary layer bits. In the case of 4/16-QAM hierarchical modulation, when $\lambda=0$, the modulation scheme degenerates to QPSK while $\lambda=1$ makes it a 16QAM modulation. Both extremes have little significance in our context and we only consider the cases of $0<\lambda<1$ where basic layer bits are more robust than secondary layer bits.

\section{Multi-hop transmission with hierarchical modulation}

Let us examine how hierarchical modulation expedites packet deliveries in wireless multi-hop networks with a simple example. Figures 2 and 3 show packet forwarding scenarios without and with hierarchical modulation, respectively. The source intends to send three packets to the destination through three intermediate nodes. In these examples, we assume the two-ray ground propagation model. If a receiver is $d$ distance away from a transmitter, then the received signal strength is $c \frac{1}{d^{4}}$ where $c$ is 0.2 . We also assume that hierarchical modulation consists of one or two layers; that is a symbol consists of the basic information and one or zero secondary information. We also assume that only two relay nodes in the directions of progress can receive informations. A relay node close to the transmitter decodes all layers while a far relay node decodes only the basic layer.

If we do not use hierarchical modulation, it requires 12 transmissions to transfer three packets from the source to the destination. With hierarchical modulation, the number of transmissions reduces to 9. In the example shown 


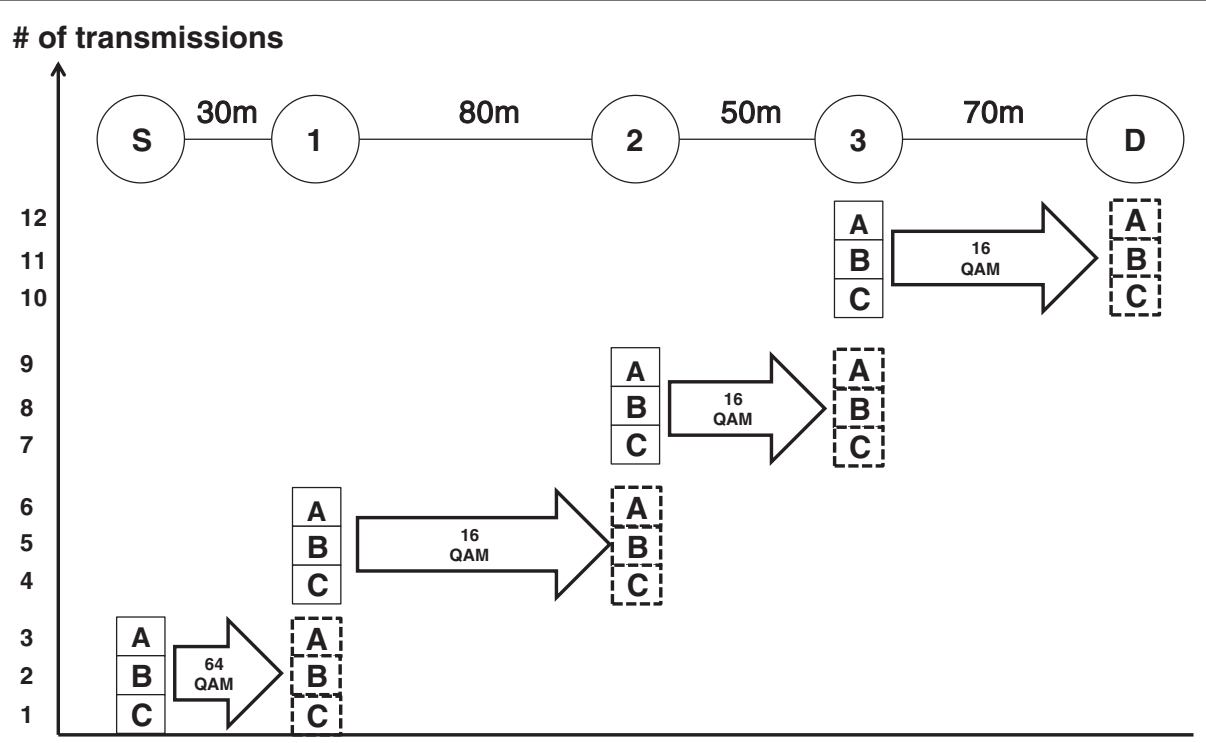

Figure 2 A scenario of non-hierarchical modulation.

in Figure 3, the SNRs of signals that the source node $s$ transmits at receivers 1 and 2 are 30.4576 and $18.3903 \mathrm{~dB}$, respectively. The source transmits packets using 4/64QAM hierarchical modulation that consists of a two bit basic-layer and a four-bit secondary layer. Assuming that packets are of the equal size, we modulate the first packet as the basic-layer and the remaining two packets as the secondary layer. Thanks to the characteristic of hierarchical modulation, the packet encoded in the basic layer can be decoded at node 2 as well as at node 1 . Thus node 1 only needs to relay the secondary layer information (i.e., the second and third packets) to node 2 . In this way, the total number of transmissions reduces from twelve to nine, enhancing the network efficiency.

\section{MDP modeling}

In this article, the goal of optimization is to minimize the end-to-end delay. We model the optimization problem as a stationary infinite-horizon MDP [20]. An MDP is described by five parameters: epochs, states, actions, rewards and state transition probabilities. Because we are to minimize the end-to-end delay, we use costs instead of rewards in this article. The decision variable is an action that selects a transmitting node and determines its hierarchical modulation scheme during the current epoch. A hierarchical modulation scheme decides the constellation sizes of the basic and secondary layers as well as the priority parameter $\lambda$ that decides the relative importance of the first and the secondary constellations.

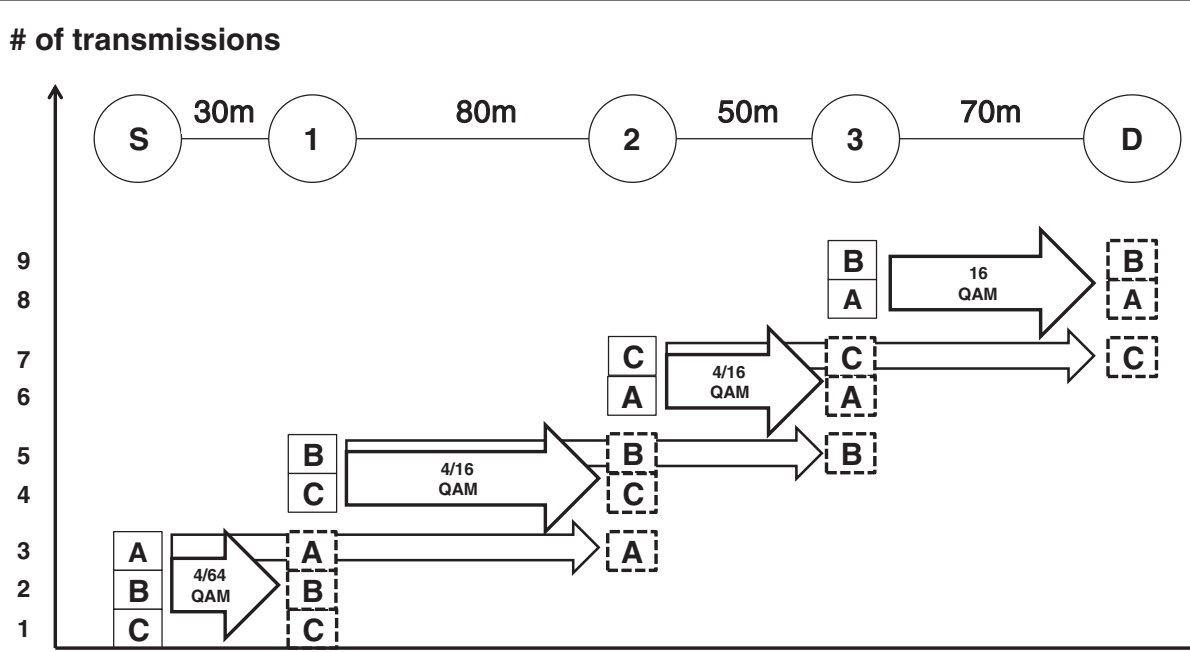

Figure $3 \mathrm{~A}$ scenario of hierarchical modulation. 


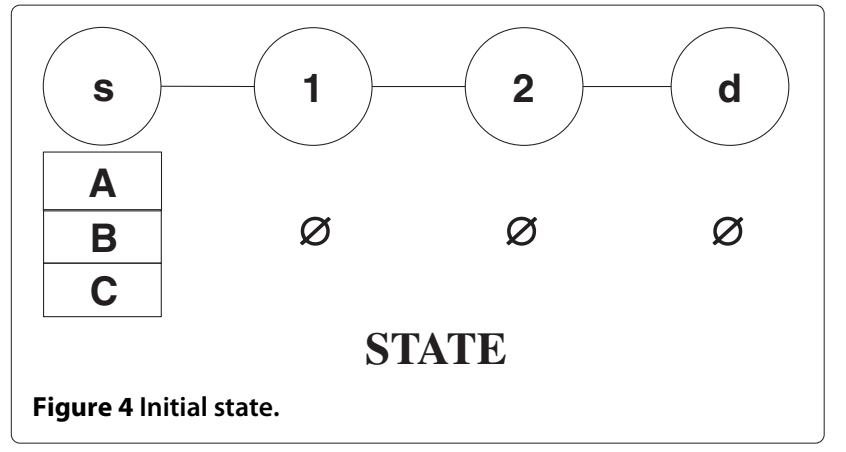

Figures 4, 5 and 6 illustrate the state and state transition example of a four-node tandem network. Figure 4 is the initial state where all three packets are in node $s$. Figure 5 shows an action that triggers a transition from state $S$ to $S^{\prime}$. The time to take an action is the packet transmission time and we use the time as the cost of the action. At the initial state, the source node will be selected as a transmitting node $t$ because it is the only node with packets. Let us assume that the transmitter uses 4/16-QAM modulation with $\lambda=0.5$. Depending on success/error deliveries of the basic and secondary information, the next states are determined as shown Figure 6. In the case of 4/64-QAM with $\lambda=0.5$, there are another 15 next states. The number of states and transitions are large even though we fix the parameter $\lambda$ at 0.5 . In reality, we should use a value between 0 and 1 .

The notations used in our model is described in Table 1. Let $\mathrm{BER}_{t, r, m(\lambda), p_{k}}$ be the bit error rate when a node $t$ transmits a packet $k$ to receiver $r$ using 4/m-QAM hierarchical modulation with the parameter $\lambda$. We employ the BER equation over AWGN channel [10]. Packet error rate, $e_{t, r, m(\lambda), p_{k}}$, can be easily derived as

$$
e_{t, r, m(\lambda), p_{k}}=1-\left(1-\mathrm{BER}_{t, r, m(\lambda), p_{k}}\right)^{h}
$$

where $h$ is the packet size in bits. Let us compute the state transition probability from $S$ to $S^{\prime}$ when $t$ is the transmitter. Let the transmitter sends packets using 4/m-QAM hierarchical modulation with the priority parameter $\lambda$. Note that intended near and far receivers are $r_{1}^{t}$ and $r_{2}^{t}$, respectively. Also let $p_{1}$ and $p_{2}$ be the basic and secondary packets, respectively. Note that $p_{1}, p_{2} \in \mathbb{K}_{S, t}$ and assume that $p_{1} \notin \mathbb{K}_{S^{\prime}, r_{1}^{t}} \cup \mathbb{K}_{S^{\prime}, r_{2}^{t}}$ and $p_{2} \notin \mathbb{K}_{S, r_{1}^{t}} \cup \mathbb{K}_{S^{\prime}, r_{2}^{t}}$. Suppose the state $S^{\prime}$ satisfies the following conditions.

$$
p_{1} \notin \mathbb{K}_{S, t}, p_{2} \notin \mathbb{K}_{S, t}, p_{1} \in \mathbb{K}_{S, r_{1}^{t}}, p_{1} \in \mathbb{K}_{S, r_{2}^{t}} \text {, and } p_{2} \in \mathbb{K}_{S^{\prime}, r_{2}^{t}}
$$

This condition specifies that $p_{1}$ is successfully delivered to two intended receivers and $p_{2}$ is also successfully delivered to the near receiver. Depending on the packets that exists at receiver nodes in state $S^{\prime}$, we derive $c_{t, r, m(\lambda), p_{k}, S^{\prime}}$, the probability of a packet $p_{k}$ is successfully (or unsuccessfully) delivered to a receiver $r$, as

$$
c_{t, r, m(\lambda), p_{k}, S^{\prime}}= \begin{cases}e_{t, r, m(\lambda), p_{k}} & p_{k} \in \mathbb{K}_{S^{\prime}, r} \\ 1-e_{t, r, m(\lambda), p_{k}} & p_{k} \notin \mathbb{K}_{S^{\prime}, r}\end{cases}
$$

Now we can define $P_{S, t, r_{1}^{t}, r_{2}^{t}, m(\lambda), S^{\prime}}$, the probability that state $S$ becomes state $S^{\prime}$ when transmitter $t$ sends packets to near receiver $r_{1}^{t}$ and far receiver $r_{2}^{r}$ using a hierarchical modulation scheme of $4 / m$-QAM and $\lambda$, as

$$
P_{S, t, r_{1}^{t}, r_{2}^{t}, m(\lambda), S^{\prime}}=c_{t, r_{1}^{t}, m(\lambda), p_{1}, S^{\prime}} \cdot c_{t, r_{2}^{t}, m(\lambda), p_{1}, S^{\prime}} \prod_{k=2}^{\frac{\log _{2} m}{2}} c_{t, r_{1}^{t}, m(\lambda), p_{k}, S^{\prime}}
$$

As shown in Equation 4, the state transition probability is composed of three factors. The first and second factors explain the delivery result of the base layer packet at near receiver $r_{1}^{t}$ and at far receiver $r_{2}^{t}$ in state $S^{\prime}$. The third factor specifies the delivery state of secondary layer packet \# 1 and 2 at the near receiver $r_{1}^{t}$ in state $S^{\prime}$.

\section{Cost (Transmission time)}

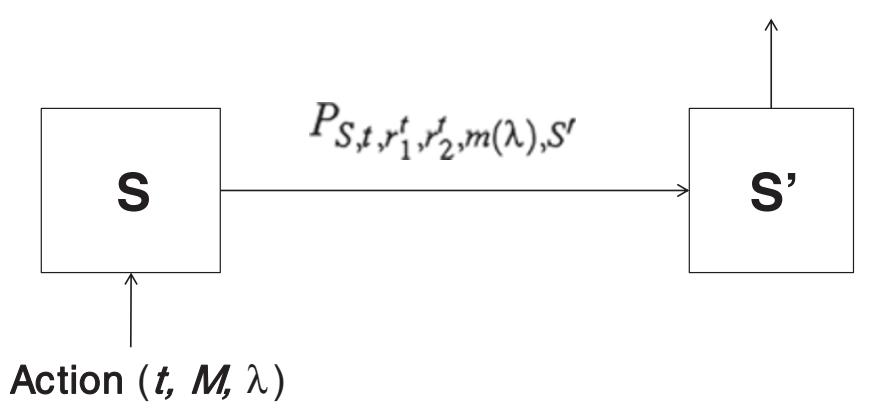

Figure 5 Action, state transition, and cost. 


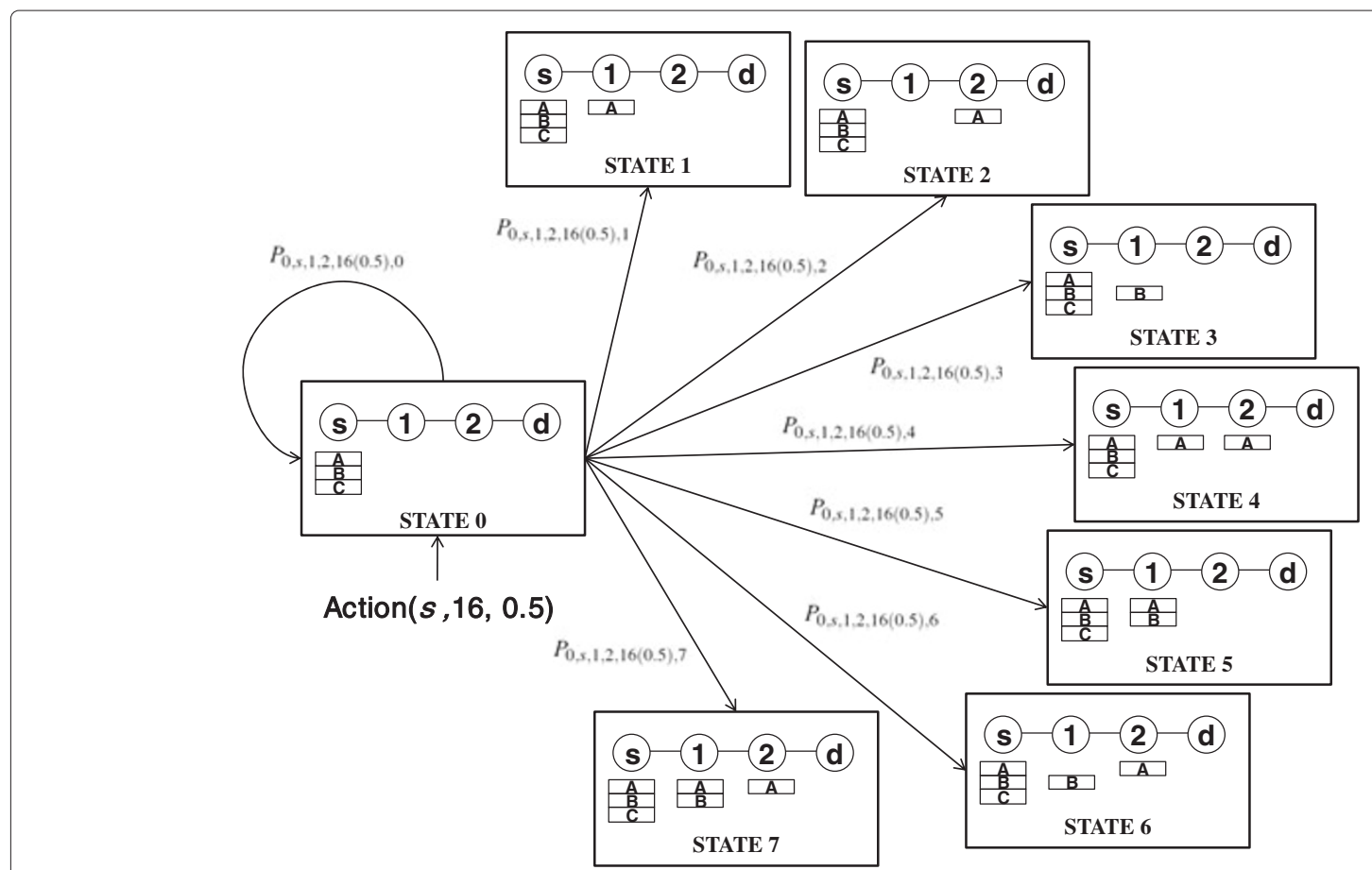

Figure 6 Next states from the initial state.

We may solve the MDP using dynamic programming in which a recursive process finds the optimal decision that minimizes total expected transmission time. Expected transmission time $T_{S}$ from current state to the final state where all packets are delivered to the final destination is iteratively calculated and the minimum value can be obtained. Following Equation 5 and 6 describe such iterative process similar with the solution in [26].

$$
T_{S}=\min _{m(\lambda), t} \frac{L / r_{m}+\sum_{S^{\prime} \subset S} P_{S, t, r_{1}^{t}, r_{2}^{t}, m(\lambda), S^{\prime}} T_{S^{\prime}}}{1-P_{S, t, r_{1}^{t}, r_{2}^{t}, m(\lambda), S}}
$$

Table 1 Notations

\begin{tabular}{cl}
\hline Series & Parameter \\
\hline$S$ & current state \\
$S^{\prime}$ & next state \\
$\mathbb{K}_{S, i}$ & a set of packets stored in node i at State $S$ \\
$m$ & $4 / m$-QAM hierarchical modulation $(m=16$, \\
& $m=64)$ \\
$\lambda$ & constellation priority parameter \\
$p_{k}$ & packet index (1: basic layer, $2:$ \\
& secondary layer \# $1,3:$ secondary layer \# 2) \\
$t$ & transmitter \\
$r_{1}^{t}$ & receiver close to the transmitter \\
$r_{2}^{t}$ & receiver far from the transmitter \\
\hline
\end{tabular}

$$
\{m(\lambda), t\}^{*}=\arg \min _{m(\lambda), t} \frac{L / r_{m}+\sum_{S^{\prime} \subset S} P_{S, t, r_{1}^{t}, r_{2}^{t}, m(\lambda), S^{\prime}} T_{S^{\prime}}}{1-P_{S, t, r_{1}^{t}, r_{2}^{t}, m(\lambda), S}}
$$

\section{Heuristic}

Three node subgraph approximation

In the MDP problem formulated in the previous section, the number of states is $O\left(N^{P}\right)$ where $N$ is the number of nodes and $P$ is the number of packets. To find the optimal solution, we need to use iterative dynamic programming whose complexity increases exponentially in terms of the number of nodes and packets. Therefore, it is impossible to find the optimal actions when the number of nodes and packets are reasonably large. For that reason, we propose a heuristic solution.

The complexity of the MDP problem in mesh networks [21] stems from the fact that the problem considers routing and hierarchical modulation at the same time. We device a novel heuristic scheme that separates routing and hierarchical modulation problems. To separate the problems, we concentrate on a subgraph consists of a transmitter and two receivers. From a original graph, we select a three node subgraph that has some potential to harvest the benefits of hierarchical modulation. For a given subgraph, we determine an optimal hierarchical modulation scheme that selects $\mathrm{m}$ in $4 / \mathrm{m}$-QAM hierarchical modulation and parameter $\lambda$. The optimality criterion is the expected time to complete the intended hierarchical 
modulated transmission. Figure 7 illustrates the decision process in a three-node subgraph. Mathematically, this procedure is denoted as

$$
\begin{aligned}
m(\lambda)^{*}= & \arg \min _{m(\lambda)}\left(\left(\frac{1}{1-e_{t, r_{1}^{t}, m(\lambda), p_{1}}}+\frac{1}{1-e_{t, r_{1}^{t}, m(\lambda), p_{2}}}\left(\frac{\log _{2} m}{2}-1\right)\right) L / R_{m}\right. \\
& \left.+\left(e_{t, r_{2}^{t}, m(\lambda), p_{1}}\right)^{\frac{1}{1-e_{t, r_{1}^{t}, m(\lambda), p_{1}}}} \frac{1}{1-e_{r_{1}^{t}, r_{2}^{t}, m_{1,2}, p_{2}}} L / R_{m_{1,2}}\right) /\left(\frac{\log _{2} m}{2}+1\right)
\end{aligned}
$$

The suggest heuristic has the same intuition as the MDP in that it finds the appropriate hierarchical modulation with the parameter $\lambda$ looking at future state. However the heuristic solution partitions the graph with subgraphs of three nodes and considers each of them as an independent problem. Figure 7 illustrates this environment. When a sender wish to send packets to near receiver and far receiver using 4/16-QAM hierarchical modulation, it is desirable for the near receiver to receive two packets and for the far receiver to receive one packet. The metric derived in Equation 7 is the expected transmission time from the current state till such the desirable state.

Contrary to tandem networks where paths are already determined, in mesh networks, we must select the paths that best realize the potential benefits of hierarchical modulation. Existing Routing metrics like ETX [22], ETT [23] only consider link quality, bandwidth and packet size but are not aware of hierarchical modulation. We need to introduce a new metric that takes the effect of hierarchical modulation into consideration. It turns out that we may not be able to invent the new metric without convert the original graph.

We apply the same three node subgraph approximation to the routing problem in mesh networks. Given an original graph, we select any three connected nodes to form a subgraph. We apply the same heuristics explained above to the subgraph. Let $t, r_{1}$, and $r_{2}$ be a transmitter, near receiver and far receivers, respectively. From a three-node subgraph, we construct a virtual subgraph as shown in Figure 8. An edge in the original graph becomes a node in the virtual subgraph and the near receiver becomes an

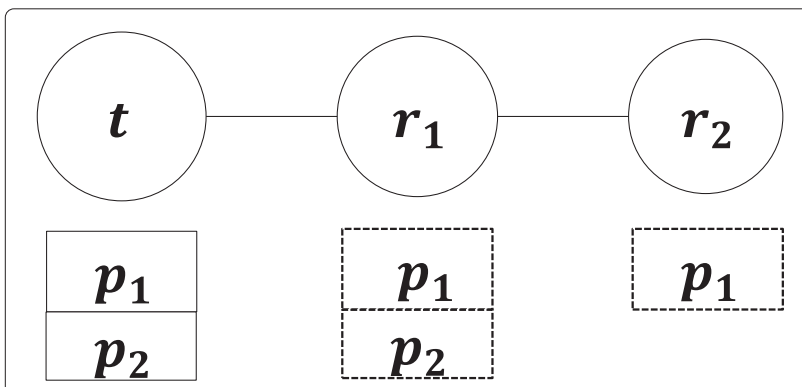

Figure 7 Intended result of hierarchically modulated transmission.

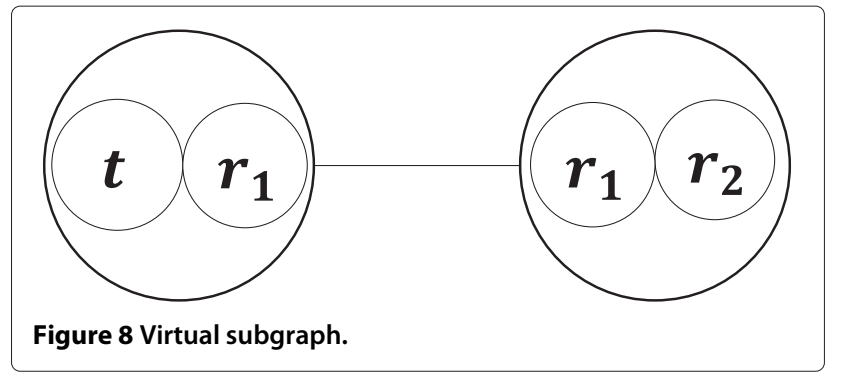

edge. The cost of the virtual edge is the expected time to finish the intended hierarchical modulated transmission that we computed in Equation 7.

There are at most $N^{3}$ three node subgraphs. We connect two subgraphs if they have a common virtual node. Therefore there are at most $N^{3}$ nodes and $N^{3}$ edges in the virtual subgraph. Figure 9 is an example of a virtual subgraph mapped from the original graph in Figure 10. After mapping the original graph to the virtual subgraph, we can easily find the shortest path from source to destination applying one of shortest path algorithms [24].

\section{Evaluation}

We conduct performance evaluation via computer simulations. MATLAB is used as our simulators. In all simulations, we assume two-ray ground propagation model from which we extract SNR depending on locations of nodes. This SNR value is used to compute BER or PER according to the equation given in [10]. For the simplicity, we assume that the sizes of packets generated from senders are the same at 8000 bytes. The modulation schemes and the values of the parameter $\lambda$ used in our simulation are shown in Table 2.

Two types of network topology are used in our study. One type is a tandem network and the other is a random mesh network. Note that it takes much time to search for exact MDP solutions in mesh networks. To obtain exact solutions, we use simple tandem networks where routing is not necessary. In a tandem network, distances between adjacent nodes are randomly assigned from a range [30, 120] $\mathrm{m}$.

We compare four transmission and routing schemes. One scheme referred to as $\mathrm{HM}$ (Heuristic) is the proposed heuristic technique. $\mathrm{HM}$ (fixed) first selects end-to-end paths based on the ETX metric and applies hierarchical modulation upon the paths. The parameter $\lambda$ is fixed at 0.5 in $\mathrm{HM}$ (fixed). $\mathrm{HM}$ (variable) is similar to $\mathrm{HM}$ (fixed) in selecting end-to-end paths. However, HM(variable) selects the best value of the parameter $\lambda$ for hierarchical modulated transmissions. Non-HM uses the ETX metric to select end-to-end paths and use non-hierarchical modulation during packet transmission. In addition to above 


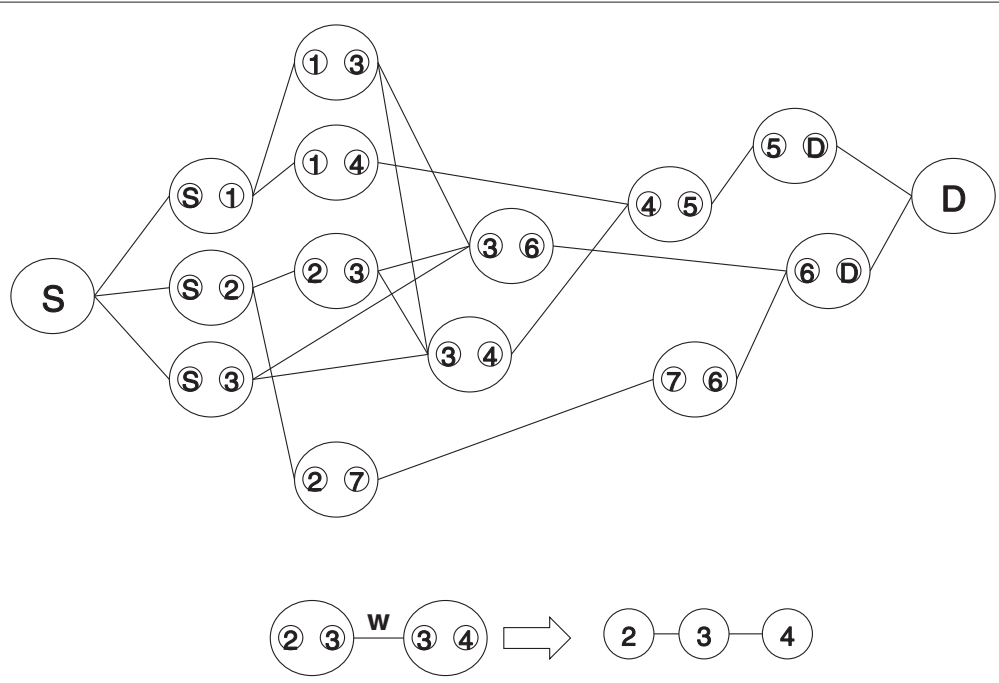

Figure 9 Reconstructed topology.

four schemes, the exact solution of the MDP problem is referred to as $\mathrm{HM}(\mathrm{MDP})$.

Figures 11 and 12 show average throughputs of four schemes in tandem networks composed of four and five nodes, respectively. We randomly generate 100 tandem topologies and measure the average and $5 \%$ confidence intervals from 100 runs. We can observe similar performance patterns in both graphs. In both four node and five node cases, the exact MDP solutions enjoy the highest throughput. Even though the throughput of our heuristic scheme is lower than that of exact solutions, the difference is relatively small at about $5 \%$. There is

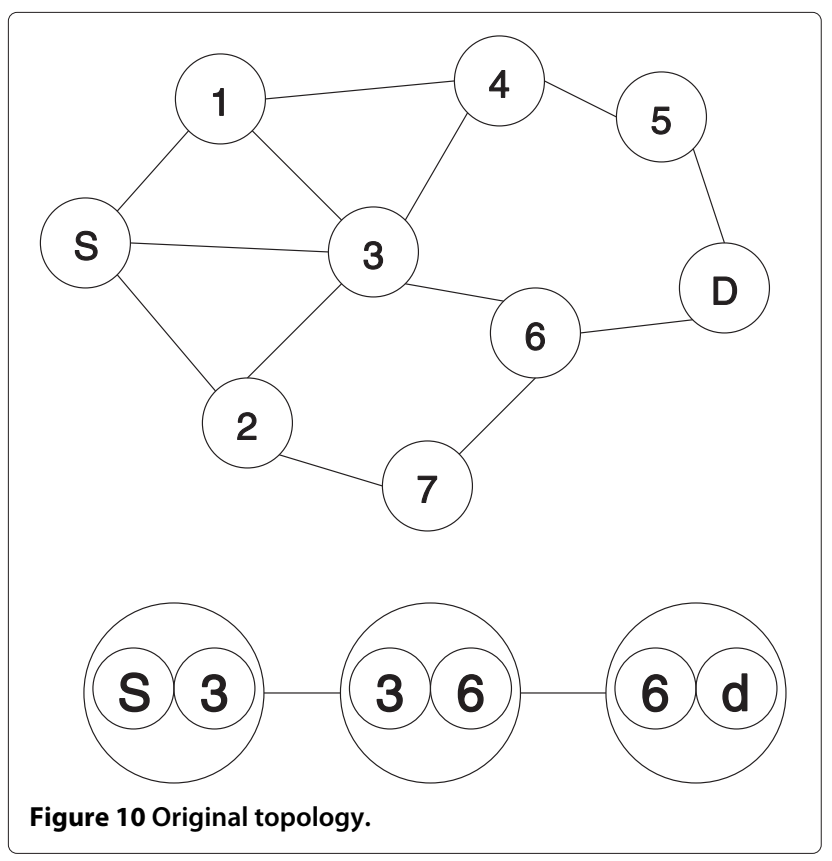

an important thing we have to discuss. The performance of HM(fixed) is sometimes lower than that of Non-HM despite of using hierarchical modulation. Because $\lambda$ is fixed in $\mathrm{HM}$ (fixed), the probability of failed hierarchical modulated transmissions can be quite large and this degrades the performance of $\mathrm{HM}$ (fixed). It is worthwhile to note that $\mathrm{HM}$ (Heuristic) perform about $20 \%$ better than HM(fixed) implying the effectiveness of the heuristic scheme.

Figures 13 and 14 illustrate the performance of three schemes in randomly generated mesh networks. We omit $\mathrm{HM}(\mathrm{MDP})$ because it is time consuming to obtain the exact optimal solution. We vary the number of nodes in a network to investigate the effects of path length on the performance. Figures 13 and 14 depict the throughput as a function of the number of flows. As the number of flows increases, the workload levied to networks also grows. The throughput of all three schemes increases as the number of flows increase up to four flows. However, all three schemes suffer from increased interference and their performance degrades when the number of flows is greater than four. We can also observe that

Table 2 Modulation schemes and $\lambda$ used in simulations

\begin{tabular}{cccc}
\hline Modulation scheme & $\lambda$ & Modulation scheme & $\lambda$ \\
\hline BPSK & - & QPSK & - \\
4/16 QAM & 0.2 & 4/64 QAM & 0.2 \\
4/16 QAM & 0.4 & 4/64 QAM & 0.4 \\
4/16 QAM & 0.6 & 4/64 QAM & 0.6 \\
4/16 QAM & 0.8 & 4/64 QAM & 0.8 \\
16 QAM & - & 64 QAM & - \\
\hline
\end{tabular}




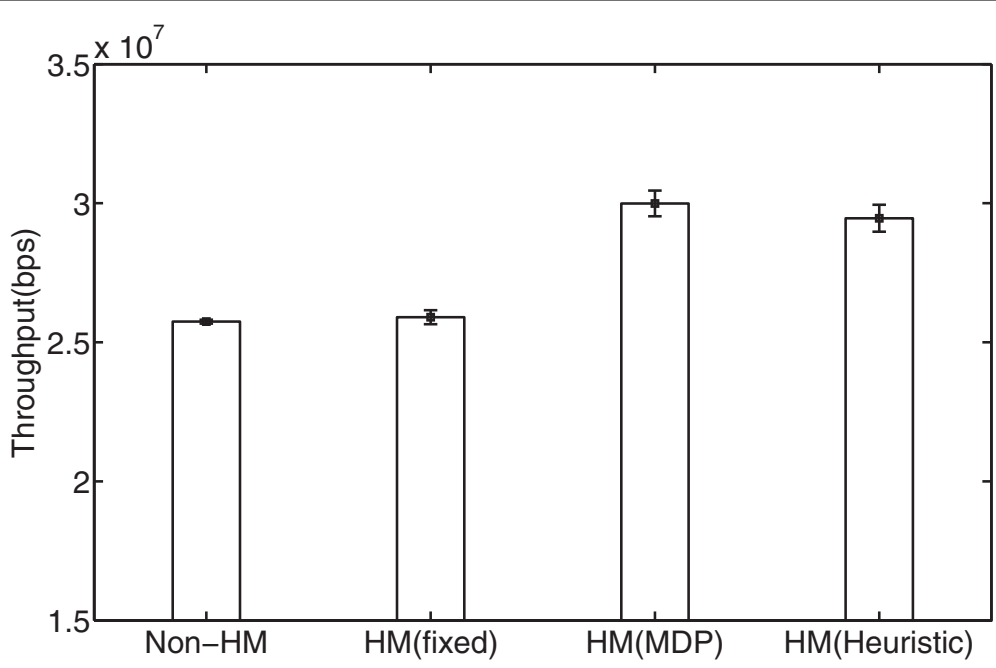

Figure 114 node.

the performance of 30 node networks is slightly better than that of 20 node networks. In both Figures 13 and 14, we observe that $\mathrm{HM}$ (Heuristic) performs best among three schemes. $\mathrm{HM}$ (Heuristic) is about $5 \%$ better than that of $\mathrm{HM}$ (variable) when there are more than three flows and about $25 \%$ better than that of NonHM(ETX). HM(variable) performs about $20 \%$ better than Non-HM(ETX) in mesh networks.

Additionally, we studied randomly generated mesh networks when the assumptions of AWGN channels and universally used two-ray ground propagation model are broken. In order to simulate the real wireless channel reflecting shadowing and multi-path effects, log-normal shadowing model is employed. Path loss exponent and standard deviation for this model are 2.5 and $5 \mathrm{~dB}$ [25].
Figures 15 and 16 show throughput results in this real wireless environment. Although overall throughput becomes lower due to fading caused by shadowing and multi-path effects, $\mathrm{HM}$ (Heuristic) records the highest throughput and has the same tendency in Figures 13 and 14.

\section{Conclusions}

This article deals with unicast communications with hierarchical modulation in multi-hop wireless network. Most previous study that deals with unicasting with hierarchical modulation assumes networks of three nodes-that is a source, an intermediate node and a destination. This article lifts the restriction and investigates hierarchical modulation in general multi-hop wireless networks. In mesh

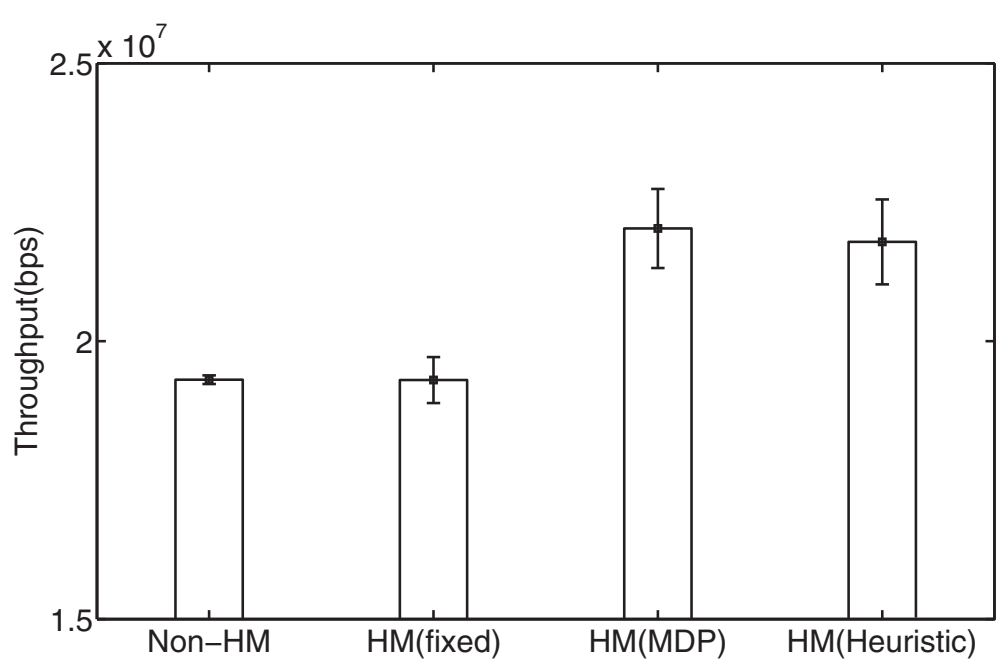

Figure 125 node. 


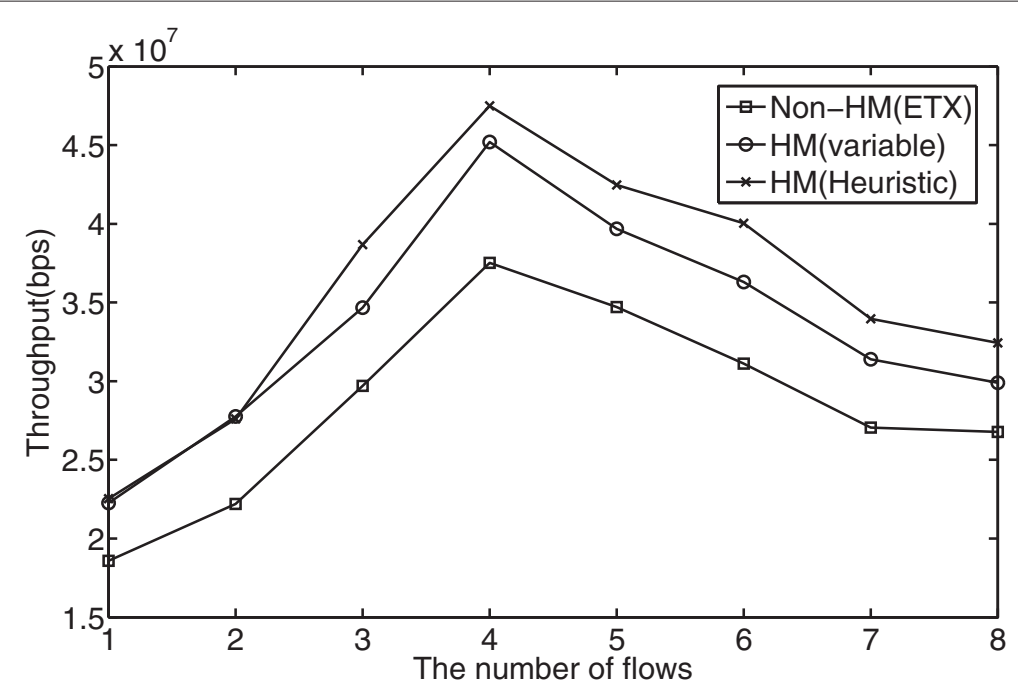

Figure 1320 node.

network, we must handle routing as well as hierarchical modulation. The optimization problem is to find the most efficient end-to-end path that realizes the potential benefits of hierarchical modulation. We model the optimization problem using a stationary infinite-horizon MDP. An action in this MDP problem determines a transmitter and a hierarchical modulation scheme. A hierarchical modulation scheme decides basic and secondary constellations and the priority parameter $\lambda$. However, due to the high complexity of the MDP problem, if we use dynamic programming to solve the MDP problem, we get the exact solutions of the MDP only for very small networks. Therefore we devise a novel scheme that separates routing and hierarchical modulation. From the original graph, we pick a subgraph with a transmitter and two receivers. For each three node subnetwork, we select the best hierarchical modulation scheme that optimally transfers packets from the transmitter to the two receivers. After we find the best hierarchical modulation schemes for all possible subnets, we map a original network into a virtual network. Once virtual network mapping is completed, we then easily find the shortest path from a source to a final destination applying one of shortest path algorithms. We carry out vigorous performance study using MATLAB simulations. Our results show that hierarchical modulation enhances the throughput about 25-30\% compared

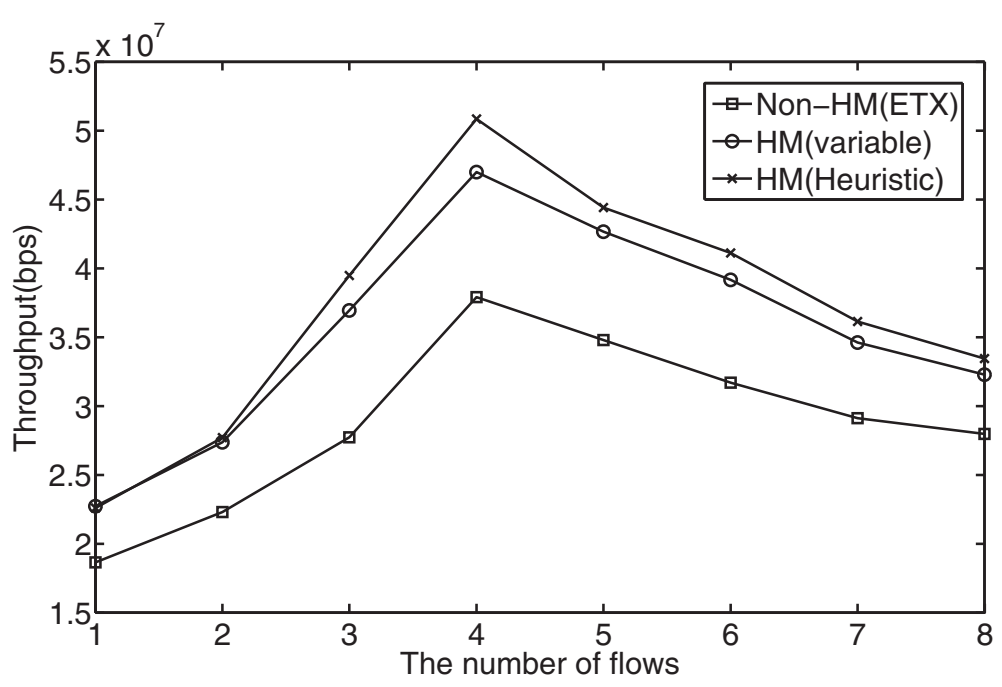

Figure 1430 node. 


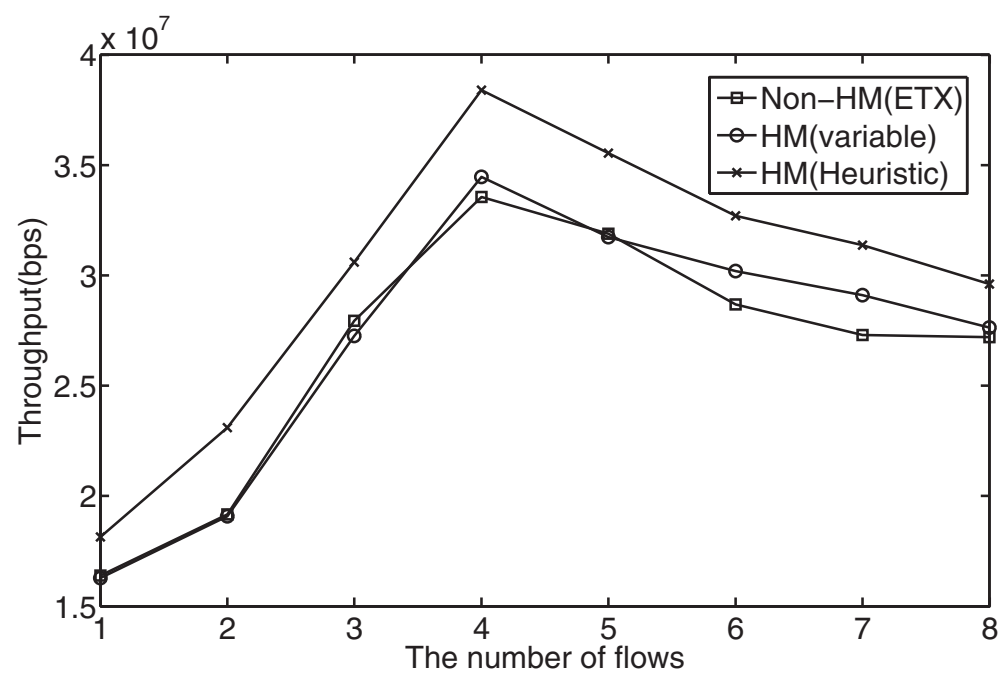

Figure 1520 node (log normal shadowing).

to non-hierarchical modulation. However, optimal solution derived by solving the MDP is also $5 \%$ better than heuristic solutions, suggesting the necessity to develop better heuristics in future. Furthermore, our study is being expanded to take multi-channel into account when finding the most profitable end-to-end path and we are planning to experiment our schemes instead of simulations.

\section{Methods}

We model $\lambda$ and modulation selection process as a stationary infinite-horizon MDP. MDPs are useful to model sequential decision making finding out a decision that maximizes profits. MDP model can be described with five features: decision epochs, states, actions, rewards (costs) and state transition probabilities. At each decision epoch in process, there is a state that reflects current system. There are candidate actions in terms of current state and the decision maker chooses one of them. The action makes MDP move into the next decision epoch and change the state into possibly different state according to state transition probabilities. The decision also receives reward (cost) resulting from the action. At each decision epoch, the same things happen and the decision maker continuously receives rewards (costs) cumulatively. These cumulative rewards (costs) are dependent on a series of actions which is called policy. The objective of MDPs is to choose the policy that maximizes rewards or minimizes costs. This process is Markov since the reward and the

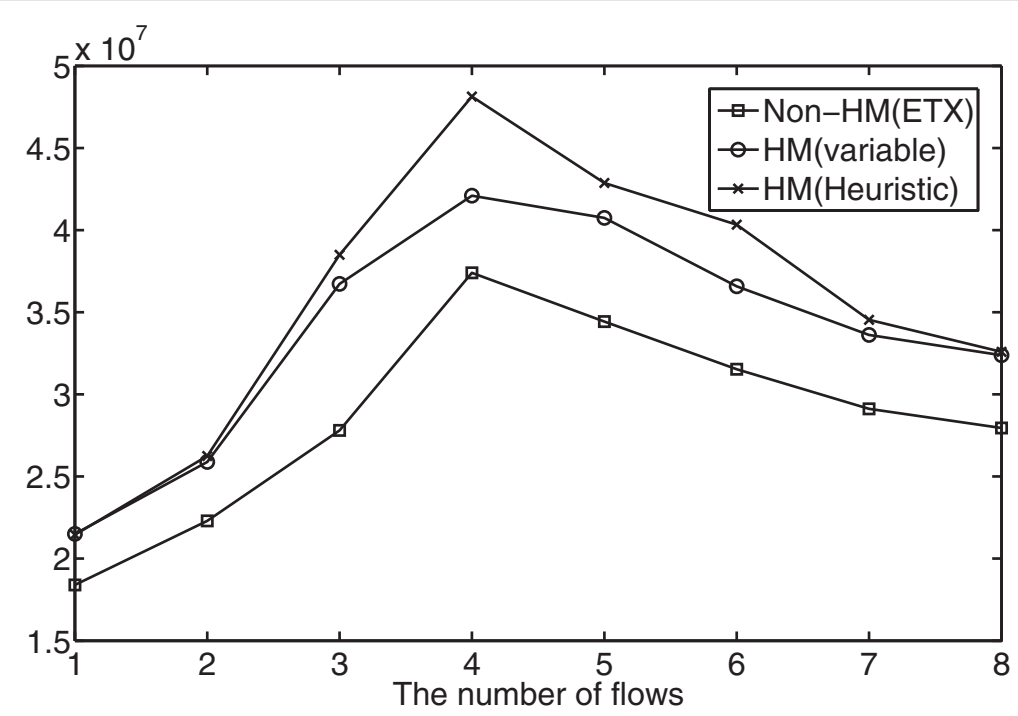

Figure 1630 node (log normal shadowing). 
transition probability are dependent on the current state and action the decision maker takes but irrespective of any previous state or action.

\section{Endnotes}

a There are at most $N^{3}$ subnets where $N$ is the number of nodes.

\section{Competing interests}

The authors declare that they have no competing interests.

\section{Acknowledgements}

This study was supported by the Korea Science and Engineering Foundation (KOSEF) grant funded by the Korea government (MEST) (No. 20100027410). This study was supported by Seoul R\&BD Program (PA100062M0209722).

Received: 8 January 2012 Accepted: 17 July 2012

Published: 31 July 2012

\section{References}

1. H Jiang, PA Wilford, A hierarchical modulation for upgrading digital broadcast systems. IEEE Trans. Broadcast. 51(2), 223-229 (2005)

2. MB Pursley, SJ M, Nonuniform phase-shift-key modulation for multimedia multicast transmission in mobile wireless networks. IEEE J. Sel. Areas Commun. 17(5), 774-783 (1999)

3. MJ Hossain, PK Vitthaladevuni, MS Alouini, VK Bhargava, AJ Goldsmith, Adaptive hierarchical modulation for simultaneous voice and multiclass data transmission over fading channels. IEEE Trans. Veh. Technol. 55(4), 1181-1194 (2006)

4. H Jiang, PA Wilford, SA Wilkus, Providing local content in a hybrid single frequency network using hierarchical modulation. IEEE Trans. Broadcast. 56(4), 532-540 (2010)

5. Digital video broadcasting (DVB): Framing structure, channel coding and modulation for digital terrestrial television. Baseline system. ETSI EN 300 744 v1.6.1. (2009)

6. MR Chari, F Ling, A Mantravadi, FLO physical layer: an overview. IEEE Trans. Broadcast. 53, 145-160 (2007)

7. Physical layer for ultra mobile broadband (UMB) air interface specification. 3GPP2 C.S0084-001-0 v2. (2007)

8. J Lee, H Kim, J Kim, B Koo, N Eum, H Lee, Design of AT-DMB baseband receiver SoC. ETRI J. 31(6), 795-802 (2009)

9. TValery, V Moeyaert, S Bette, P Megret, Performance analysis of DVB-H OFDM hierarchical modulation in impulse noise environment. in 2007 14th IEEE Symposium on Communications and Vehicular Technology in the Benelux (Delft University of Technology Delft, Netherlands, 2007), pp. 1-4

10. PK Vitthaladevuni, MS Alouini, BER computation of 4/M-QAM hierarchical constellations. IEEE Trans. Broadcast. 47(3), 228-239 (2001)

11. P Vitthaladevuni, MS Alouini, A recursive algorithm for the exact BER computation of generalized hierarchical QAM constellations. IEEE Trans. Inf. Theory. 49, 297-307 (2003)

12. MK Chang, SY Lee, Performance analysis of cooperative communication system with hierarchical modulation over rayleigh fading channel. IEEE Trans. Wirel. Commun. 8(6), 2848-2852 (2009)

13. C Hucher, P Sadeghi, Hierarchical modulation-based cooperative scheme: minimizing the symbol error probability. in 2010 IEEE 17th International Conference on Telecommunications (ICT) (Doha, 2010), pp. 309-315

14. C Hausl, J Hagenauer, Relay communication with hierarchical modulation. IEEE Commun. Lett. 11, 64-66 (2007)

15. K Lee, $\mathrm{D}$ Cho, Hierarchical constellation based adaptive relay scheme in multi-hop networks. IEEE Commun. Lett. 11(3), 225-227 (2007)

16. H Son, J Park, L S, Hierarchical modulation-based cooperation utilizing relay-assistant full-duplex diversity. Wirel. Netw. 17(3), 583-595 (2010)

17. RJ Whang, L Huaping, EK Hong, Multiuser cooperative relay communication employing hierarchical modulation. in 2010 IEEE 71 st Vehicular Technology Conference (VTC 2010-Spring) (Taipei, 2010), pp. 1-5

18. HX Nguyen, HH Nguyen, T Le-Ngoc, Signal transmission with unequal error protection in wireless relay networks. in IEEE Global Telecommunications Conference (Honolulu, HI, 2009), pp. 1-6
19. A Annamalai, L Jing, DR Vuman, A receiver-oriented rate-adaptation strategy for improving network efficiency in mobile ad-hoc networks. in IEEE Communications Society: Sarnoff Symposium (Princeton, NJ, 2008), pp.1-5

20. ML Puterman, Infinite-Horizon Model. in Markov Decision Processes: Discrete Stochastic Dynamic Programming, (New Haven, Wiley-Interscience, 1994), pp. 119-141

21. IF Akyildiz, X Wang, W Wang, Wireless mesh networks: a survey. Comput. Netw. ISDN Syst. 47(4), 445-487 (2005)

22. DSJ De Couto, D Aguayo, J Bicket, R Morris, A high-throughput path metric for multi-hop wireless routing. in Proceedings of the 9th annual international conference on Mobile computing and networking (San Diego, California, ACM, 2003), pp. 134-146

23. R Draves, J Padhye, B Zill, Routing in multi-radio, multi-hop wireless mesh networks. in Proceedings of the 10th annual international conference on Mobile computing and networking (Philadelphia, Pennsylvania, ACM, 2004), pp. 114-128

24. TH Cormen, CE Leiserson, RL Rivest, C Stein, Single-source shortest paths. in Introduction to Algorithms, 2nd edn. (Cambridge, Massachusetts, MIT Press, 2001), pp. 580-619

25. LC Liechty, Path loss measurements and model analysis of a $2.4 \mathrm{GHz}$ wireless network in an outdoor environment. Master's Thesis, Georgia Institute of Technology (2007)

26. X Zhao, J Guo, T CC, A Misra, S Jha, A high-throughput routing metric for reliable multicast in multi-rate wireless mesh networks, in 2011 Proceedings IEEE INFOCOM. (Shanghai, 2011), pp. 2042-2050

doi:10.1186/1687-1499-2012-240

Cite this article as: Park et al:: Multi-hop transmission and routing with hierarchical modulation. EURASIP Journal on Wireless Communications and Networking 2012 2012:240.

\section{Submit your manuscript to a SpringerOpen ${ }^{\circ}$ journal and benefit from:}

- Convenient online submission

- Rigorous peer review

- Immediate publication on acceptance

- Open access: articles freely available online

- High visibility within the field

- Retaining the copyright to your article

Submit your next manuscript at $\boldsymbol{\nabla}$ springeropen.com 